Article

\title{
Size Separation of Silica Particles Using a Magnetite-Containing Gel-Packed Column
}

\author{
Manoka Miyoshi ${ }^{1}$, Kosuke Takayanagi ${ }^{1}$, Shintaro Morisada ${ }^{1}$, Keisuke Ohto ${ }^{1}$,

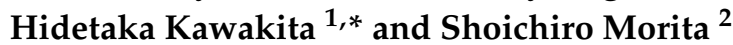 \\ 1 Department of Chemistry and Applied Chemistry, Saga University, Saga 840-8502, Japan; \\ 17578033@edu.cc.saga-u.ac.jp (M.M.); kesuko.1203@outlook.com (K.T.); morisada@cc.saga-u.ac.jp (S.M.); \\ ohtok@cc.saga-u.ac.jp (K.O.) \\ 2 Nitchitsu Co., Ltd., Nagasaki 859-6203, Japan; s-morita@nitchitsu.co.jp \\ * Correspondence: kawakita@cc.saga-u.ac.jp; Tel.: +81-952-28-8670
}

Received: 23 February 2019; Accepted: 4 April 2019; Published: 8 April 2019

check for updates

\begin{abstract}
A magnetite-containing gel was prepared by water-in-oil radical polymerization of $N, N$-dimethylacrylamide and $N, N^{\prime}$-methylenebisacrylamide in the presence of magnetite. The size of the prepared gel particles was $86 \mu \mathrm{m}$. The obtained magnetite-containing gel was packed in a column and first permeated with water, which revealed that the gel displayed a nonlinear response to pressure drop with increasing flow rate. Thus, the gel particles at the bottom of the column felt more pressure from the fluid than those at the top, causing greater deformation of the gel particles at the bottom of the column than at the top. The gaps between the packed gel particles functioned as pores to filter particles of appropriate size and morphology. An industrial silica particle suspension with particle sizes of $300 \mathrm{~nm}, 800 \mathrm{~nm}$, and $10 \mu \mathrm{m}$ was permeated through the gel layer. The smallest $(300 \mathrm{~nm})$ silica particles passed through the column. The filtered silica particles were recovered from the gel layer by using a magnet to separate the magnetite-containing gel from the filtered silica particles. This magnetite-containing gel has wide application prospects for the separation of not only ceramics but also other colloids.
\end{abstract}

Keywords: magnetite-containing gel; separation; deformation; recovery; silica particle

\section{Introduction}

Separation of colloidal particles such as microorganisms, cells, and ceramic particles has been studied. The sophisticated separation of colloids depends on their different characteristics. For example, a porous membrane combined with chromatography has been used to separate a microorganism suspension via $\mathrm{pH}$-gradient flow through a particle-packed column [1], and microfiltration with a cross flow has been performed to sweep the filtered residue from the membrane pores [2,3]. Glass beads packed in a column and microchannels have been used for separation based on the different mobilities of the colloidal particles [4,5]. At present, the pores in a membrane, the gaps between glass beads, and the microchannel diameter used for separation need to be uniform. If the pores used for filtration were able to be dynamically change, separation performance could be greatly enhanced in a wide range of applications.

In chromatography, spherical gel particles are packed in a column, and then water is permeated through the column to increase the pressure drop by narrowing the gaps between the gel particles through the deformation of the packed gel. The gel particles at the bottom of the column feel more pressure and thus deform to a greater extent than the gel particles at the top of the column. Through such deformation, the gaps between the gel particles become smaller at the bottom of the column compared with those at the top. Because the flow of water through deformable gel particles changes 
the pores in the flow direction, injected colloids are dynamically separated depending on their size and morphology [6]. Silica particle suspensions have been separated using gel particle deformation in columns. The spherical gel particles prepared by radial polymerization were packed in a column to separate an injected silica particle suspension with particle sizes ranging from $100 \mathrm{~nm}$ to $10 \mu \mathrm{m}$ It was found that silica particles with a diameter of $100 \mathrm{~nm}$ passed through the column, whereas those with a diameter of $10 \mu \mathrm{m}$ were captured at the top of the gel layer. The dynamic filtering efficiency of silica particles with a diameter of $1 \mu \mathrm{m}$ depended on the gel size, gel elasticity, and flow rate of water. It may be possible to recover a filtered colloid at the top of the gel layer by floating the filtered colloid particles using the elasticity of the gel layer and then applying a cross flow across the column [7]. However, an appropriate technique to recover the filtered colloid particles in a gel layer has not been proposed yet.

In this study, particle separation using a magnetite-containing gel layer in a column is proposed (Figure 1). The magnetite-containing gel should be easily moved by the application of a magnetic field to allow the separation of the filtered particles in the gel layer from the gel particles. Materials including magnetite have been studied from a chemistry perspective for their preparation $[8,9]$ and applications [10]. A macroscopic magnetite assembly has been used as a filter medium for the separation of several particles and subsequent recovery by the application of a magnetic field [11,12]. Here, a column packed with a magnetite-containing gel was used to separate industrial silica particles prepared by a dry process. Large volumes of silica particles are industrially produced by dry processes; that is, silica ore is cracked to obtain electronics materials such as fillers to control heat transfer. The regulation of the size and morphology of the cracked silica particles is crucial for potential high-performance applications. This study had three main objectives: (1) the preparation of a magnetite-containing gel, (2) the individual filtration of silica particles with diameters of 1 and $10 \mu \mathrm{m}$, and (3) the filtration of industrial silica particle suspensions using the magnetite-containing gel and the recovery of the filtered silica particles from the gel layer by using a magnet. The sizes of the silica particles obtained after separation were analyzed by dynamic light scattering (DLS).

(c)

(a)

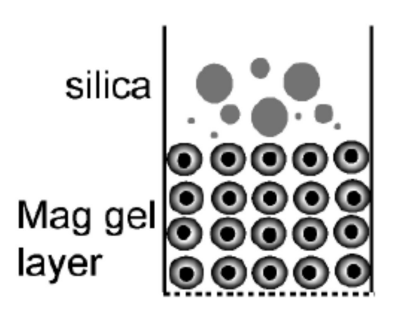

(b)

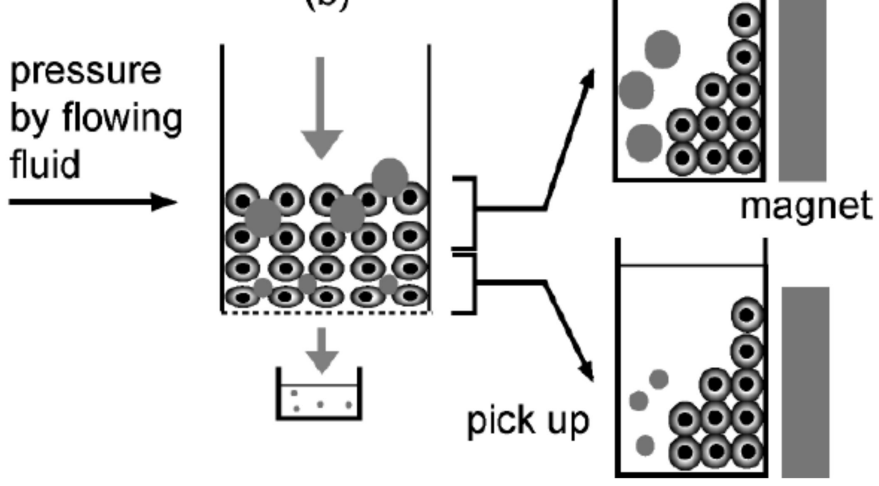

Figure 1. Separation of silica particles using a magnetite-containing gel and recovery of filtered silica particles from the gel by applying a magnet. (a) Magnetite-containing gel particles were packed in a column, and then a silica particle suspension was injected on the top of the gel layer. (b) The pressure of water flowing through the gel layer induced the deformation of the gel, which changed the filtration pores in the flow direction. As a result, small silica particles were eluted. Larger silica particles remained near the top of the column, and medium silica particles moved closer to the bottom of the column. (c) The magnetite-containing gel and filtered silica particles were taken from each region of the column, and then a magnet was applied. The magnetite-containing gel moved towards the magnet, allowing the filtered silica particles and magnetite-containing gel to be separated. 


\section{Materials and Methods}

\subsection{Materials}

Magnetite was prepared from iron (III) chloride hexahydrate (095-00875), iron (II) chloride tetrahydrate (099-00915), and sodium hydroxide (192-15985) purchased from Wako Pure Chemical Industries, Japan. D-Glucose (07-0690-6-5) was obtained from Sigma-Aldrich, MI, USA. The monomer, crosslinker, and initiator used to prepare the gel particles were $N, N$-dimethylacrylamide (049-19185), $N, N^{\prime}$-methylenebisacrylamide (M0506), and ammonium persulfate (018-03282) purchased from Wako Pure Chemical Industries, Japan. Span 80 (S0060) and Tween 80 (T2533) were obtained from Tokyo Chemical Industry Co., Japan. Ethylenediaminetetraacetic acid (EDTA; 09-1320) was purchased from Katayama Chemical Ltd., Japan. Glass beads (105-125 $\mu \mathrm{m}, \mathrm{BZ}-01)$ were purchased from AS-ONE, Japan. Black silica particles (size: $10 \mu \mathrm{m}$ ) and white silica particles (size: $1.0 \mu \mathrm{m}$ ) were purchased from Micromod Partikeltechnologie Gmbh, Germany. Industrial silica particles were donated by Nitchitsu Co., Ltd., Japan. Other chemicals were of analytical grade or higher.

\subsection{Preparation of Magnetite}

Solution 1 was first prepared by dissolving sodium hydroxide in distilled water to give a concentration of $10 \mathrm{~mol} / \mathrm{L}$. Iron(III) chloride hexahydrate $(11 \mathrm{~g}, 0.041 \mathrm{~mol})$ and iron(II) chloride tetrahydrate $(4.07 \mathrm{~g}, 0.020 \mathrm{~mol})$ were dissolved in water at $303 \mathrm{~K}$ in a water bath (Eyela, SB-350) while stirring at $500 \mathrm{rpm}$ by a stirrer (Eyela, Mazel, ZZ-1200) to give solution 2. Glucose (8.75 g, $0.049 \mathrm{~mol})$ was dissolved in solution 1 , and then solution 1 was added dropwise to solution 2 at a rate of one drop every two seconds. The obtained solution was stirred at $303 \mathrm{~K}$ and $500 \mathrm{rpm}$ for $1 \mathrm{~h}$. The precipitate present after this time responded to a neodymium magnet $(0.10 \mathrm{~T}, 50 \times 20 \times 2 \mathrm{~mm}$, Sangyo Supply Co., Ltd., Sendai, Japan). The magnetite particles were observed by optical microscopy (VH-S5, Keyence Corporation, Osaka, Japan), and their size distribution was determined by measuring the diameters of more than 200 particles. The mean size of the magnetite particles was $670 \mathrm{~nm}$.

\subsection{Polymerization of Spherical Gel Particles Including Magnetite}

To prepare the oil phase, Span 80 (9.0 g) and Tween 80 (3.0 g) were dissolved in hexane (200 g). $N, N$-Dimethylacrylamide (9.33 g), $N, N^{\prime}$-methylenebisacrylamide $(0.16 \mathrm{~g})$, EDTA $(0.15 \mathrm{~g})$, and water ( $25 \mathrm{~g}$ ) were mixed, and then ammonium persulfate $(0.33 \mathrm{~g})$ was added. The prepared magnetite suspension $(10(\mathrm{w} / \mathrm{w}) \%, 10 \mathrm{~mL})$ was dispersed in the oil phase. The water phase was dropped into the oil phase over $30 \mathrm{~s}$ and then stirred at $500 \mathrm{rpm}$ and $333 \mathrm{~K}$ for $4 \mathrm{~h}$. Under these conditions, the added magnetite particles moved into the water phase. After the polymerization, the reactor was cooled to room temperature. The obtained magnetite-containing gel suspension was filtered under vacuum and then washed with hexane $(250 \mathrm{~mL})$ and ethanol $(1 \mathrm{~L})$ twice for $2 \mathrm{~h}$. The obtained suspension was finally washed with distilled water, filtered through a nylon mesh $(161 \mu \mathrm{m}, \mathrm{NBC}$ Meshtec Inc., N-N0110S 115, P1604A09925-03, Tokyo, Japan), and then filtered again through a finer nylon mesh (42 $\mu \mathrm{m}$, NBC Meshtec Inc., N-N0330T 115, E1802A00255-01, Tokyo, Japan) to remove the residual polymer, magnetite, and larger-size gel particles. The obtained magnetite-containing gel is referred to as "Mag gel". The concentration of Mag gel in water was set to $10 \mathrm{wt} \%$. The obtained Mag gel was observed by optical microscopy (VH-S5, Keyence Corporation, Osaka, Japan), and its particle size distribution was determined by measuring the diameters of more than 200 particles. For comparison, polymerized gel spheres without magnetite were also fabricated.

The magnetite concentration in the Mag gel was quantitatively determined. Mag gel (1 mL) was dried in a drying oven (DX-31, Yamato Scientific Co., Ltd., Tokyo, Japan). Nitric acid solution $(3.0 \mathrm{M}, 25 \mathrm{~mL})$ was added to the dried gel at $333 \mathrm{~K}$, and then the mixture was agitated using a shaking bath (SB-20, As One, Japan). The time dependence of iron concentration was determined by atomic absorption spectroscopy (AA-6800, Shimadzu, Corporation, Kyoto, Japan) to determine the concentration of leaked iron ions at steady state in solution. 


\subsection{Water Permeation through the Mag Gel}

Glass beads (105-125 $\mu \mathrm{m}$ ) were first packed in a column (ID: $0.5 \mathrm{~cm}$, OD: $0.9 \mathrm{~cm}$, height: $20 \mathrm{~cm}$, 7370522, Bio-Rad Laboratories, Inc., CA, USA) to a height of $1.0 \mathrm{~cm}$. Mag gel suspension $(10 \mathrm{w} / \mathrm{w}) \%$, $1.4 \mathrm{~mL}$ ) was added to the column and then allowed to settle via gravity for $15 \mathrm{~h}$, so that the height of the gel layer was $1.0 \mathrm{~cm}$. After filling the gel layer in the column with water, water was permeated through the column at $25 \mathrm{~mL} / \mathrm{h}$ to measure the pressure drop of the Mag gel layer. The eluent was collected to check the concentrations of iron and gel that leaked from the column by ultraviolet-visible (UV-Vis) spectroscopy at $600 \mathrm{~nm}$ (V-630 BIO, JASCO, Tokyo, Japan).

\subsection{Permeation of Silica Particle Suspensions through the Mag Gel Layer}

Silica particle suspensions with particle sizes of 1.0 and $10 \mu \mathrm{m}(1.0 \mathrm{~g} / \mathrm{L}, 0.40 \mathrm{~mL})$ were individually permeated through the Mag gel-packed column at a flow rate of $25 \mathrm{~mL} / \mathrm{h}$. The eluent from the column was collected to determine the concentration of silica particles by UV-Vis spectroscopy at $600 \mathrm{~nm}$.

To separate the industrial silica particle suspension, the suspension $(1.0 \mathrm{~g} / \mathrm{L}, 0.4 \mathrm{~mL})$ injected on the top of the Mag gel layer was permeated through the column at a flow rate of 10,25 , or $50 \mathrm{~mL} / \mathrm{h}$. The eluent was collected continuously, its absorbance was determined by UV-Vis spectroscopy at $600 \mathrm{~nm}$, and the particle size was measured by DLS (ELZ-ZA PUS, Otsuka Electronics Co., Ltd., Osaka, Japan).

To recover the filtered particles in the magnetite-containing gel, the Mag gel and filtered particles in the upper half and lower half of the column were taken out by a pipette. A neodymium magnet was then applied to the bottom of each suspension to separate the filtered silica particles from the Mag gel by washing the suspension with water and ethanol. The particle size of the supernatant $(5 \mathrm{~mL})$ was determined by DLS.

\section{Results and Discussion}

\subsection{Preparation of $\mathrm{Mag} \mathrm{Gel}$}

The introduction of magnetite into the inner part of the gel spheres provided gel particles that were able to move under the application of a magnetic field. As proposed in Figure 1, after the separation of particles by Mag gel, the filtered particles could be obtained by using a magnet to remove the Mag gel particles. EDTA-adsorbed magnetite particles with a size of $670 \mathrm{~nm}$ were introduced during water/oil emulsion polymerization of $N, N$-dimethylacrylamide and $N, N^{\prime}$-methylenebisacrylamide to give the Mag gel. EDTA was adsorbed onto the magnetite particles to make their surface more hydrophilic, allowing the magnetite particles to move into the water phase during water/oil polymerization [13]. An optical microscopy image and the size distribution of the Mag gel particles are shown in Figure 2. The introduction of magnetite to the gel was confirmed, and the mean size of the Mag gel particles was $86 \mu \mathrm{m}$.

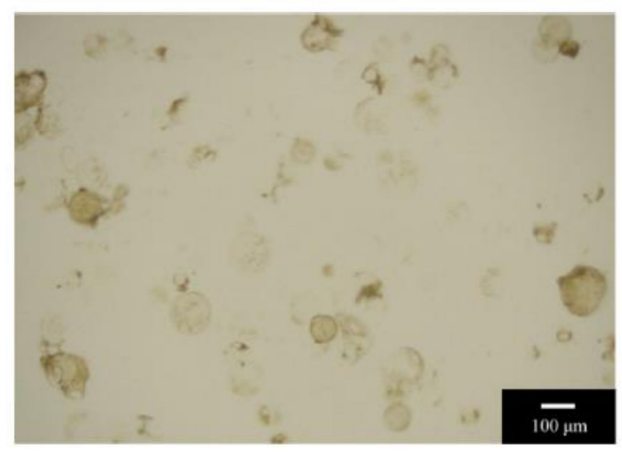

(a)

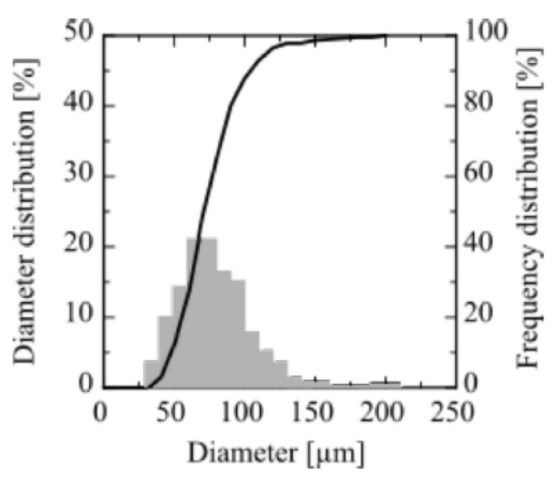

(b)

Figure 2. Optical microscopy image (a) and particle size distribution (b) of the magnetite-containing gel. 
The amount of magnetite included in the gel was determined by immersing the Mag gel in $3.0 \mathrm{M}$ $\mathrm{HNO}_{3}$ and then measuring the concentration of leaked iron ions at steady state by atomic absorption spectroscopy. From the concentration of leaked iron ions at steady state, the amount of magnetite included in the Mag gel was calculated to be $0.26 \mathrm{mg} \mathrm{Fe} / \mathrm{mg}$ dry Mag gel. For comparison, spherical gel particles without magnetite were prepared by the same method except that the magnetite particles were omitted. The magnetite-free gel particles had a mean diameter of $81 \mu \mathrm{m}$.

\subsection{Permeation of Water through Mag Gel}

The time dependence of the pressure drop during permeation of water through the Mag gel-packed column is shown in Figure 3a, along with the behavior of the reference gel-packed column (Figure 3b). No pressure drop was measured at water flow rates of 10 and $25 \mathrm{~mL} / \mathrm{h}$. However, at a flow rate of $50 \mathrm{~mL} / \mathrm{h}$, the pressure drop increased gradually over time. When water was permeated through the gel-packed column at a flow rate of $10 \mathrm{~mL} / \mathrm{h}$, as shown in Figure $3 \mathrm{~b}$, the pressure drop gradually increased and then levelled off. At the higher flow rate, the pressure drop dramatically increased in a non-linear manner. Compared with that of the Mag gel-packed column, the pressure drop of the gel-packed column was larger because of the smaller deformation of Mag gel relative to that of the reference gel in the column. This is because magnetite in the gel particles disturbed the free deformation at the contacting surfaces of the gel particles, resulting in a smaller pressure drop $[6,14]$.

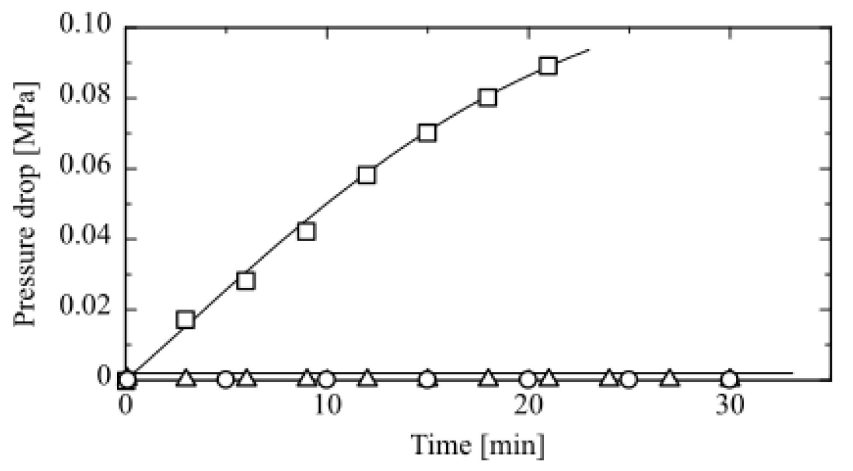

(a)

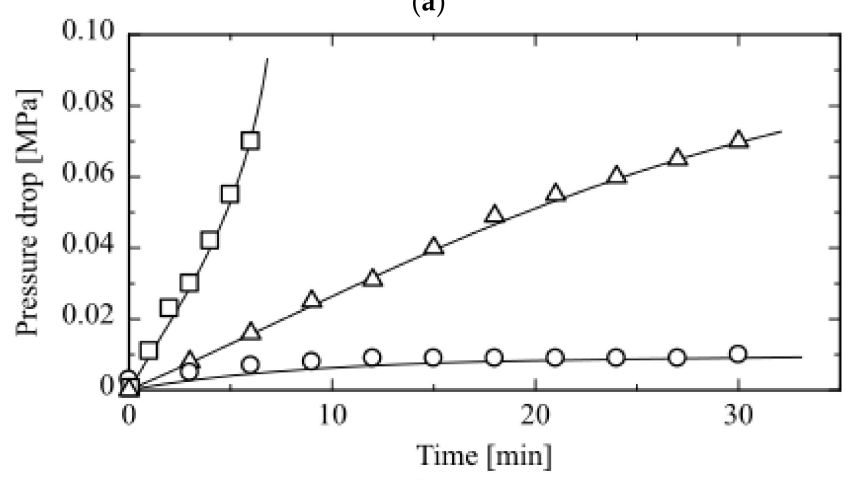

(b)

Figure 3. Time dependence of the pressure drop during permeation of water through (a) Mag gel-packed column and (b) gel-packed column. Circles: $10 \mathrm{~mL} / \mathrm{h}$, triangles: $25 \mathrm{~mL} / \mathrm{h}$, squares: $50 \mathrm{~mL} / \mathrm{h}$. The initial height of the Mag gel layer was set to $1.0 \mathrm{~cm}$.

\subsection{Permeation of Individual Silica Particle Suspensions through Mag Gel}

Silica particle suspensions with particle sizes of 1 and $10 \mu \mathrm{m}$ were individually injected on the top of the Mag gel layer, and then water was permeated through the column. The concentration and volume of each silica particle suspension were $1.0 \mathrm{~g} / \mathrm{L}$ and $0.40 \mathrm{~mL}$, respectively. The time dependence of the concentration of particles eluted from the column at a flow rate of $25 \mathrm{~mL} / \mathrm{h}$ is shown in Figure 4 . Silica particles with a diameter of $10 \mu \mathrm{m}$ eluted more slowly, and the eluted percentage was lower 
than for the $1 \mu \mathrm{m}$ particles. This is because the larger silica particles were filtered between the Mag gel particles or eluted through the column via collision with Mag gel. The elution percentages of silica particles after $30 \mathrm{~min}$ at different flow rates are summarized in Table 1. In the case of $10 \mu \mathrm{m}$ silica particles, the elution percentage was low at all flow rates. Conversely, the $1 \mu \mathrm{m}$ silica particles eluted more quickly with increasing flow rate. This is because the $1 \mu \mathrm{m}$ silica particles were transported via a convection flow of water through the Mag gel layer before the compaction of the gel layer. The balance between deformation of the Mag gel layer and transport of particles by convection through the layer should be considered to obtain appropriate separation performance. The data in Table 1 show that the Mag gel layer was able to separate particles in the size range from 1.0 to $10 \mu \mathrm{m}$.

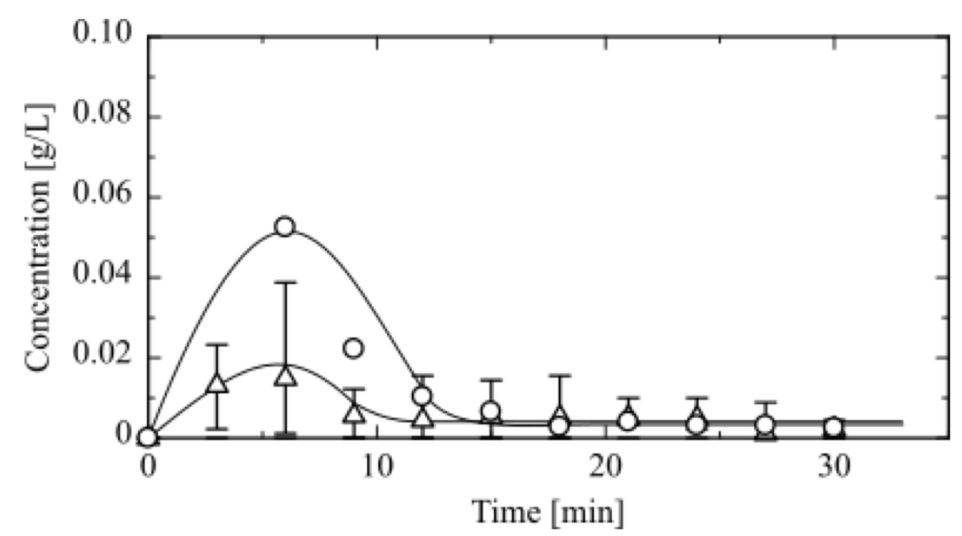

Figure 4. Time dependence of the concentration of silica particles eluted from the Mag gel-packed column. Circles: $1 \mu \mathrm{m}$, triangles: $10 \mu \mathrm{m}$. The injected volume of silica suspension was $0.4 \mathrm{~mL}$, and the suspension concentration was $1.0 \mathrm{~g} / \mathrm{L}$.

Table 1. Percentage of silica particle.

\begin{tabular}{cccc}
\hline Diameter of Silica Particle $[\boldsymbol{\mu m}]$ & \multicolumn{3}{c}{ Flow } \\
\hline & 10 & 25 & 50 \\
10 & 8 & 6 & 7 \\
1 & 55 & 50 & 63 \\
\hline
\end{tabular}

\subsection{Permeation of an Industrial Silica Particle Suspension through Mag Gel}

Silica particles are produced by wet and dry methods. When using the wet methods, although the productivity of silica is lower than that of dry methods, silica particles of uniform size may be synthesized [15]. When using the dry methods, silica ore is cracked in large quantities. However, the size and morphology of the obtained silica particles vary [16]. Obtaining uniform silica particles by a dry method is an attractive cost-effective approach with high potential for a wide range of applications. In this work, industrial silica particles produced by a dry method were provided by Nitchitsu Co., Ltd., Nagasaki, Japan An SEM image and the size distribution of these particles are shown in Figure 5. The SEM image of the silica particles indicated their various morphologies. DLS measurements showed the particle sizes in solution were $300 \mathrm{~nm}, 800 \mathrm{~nm}$, and $10 \mu \mathrm{m}$, with relative contents of $0.15,0.69$, and 0.16 , respectively.

The industrial silica particle suspension was then permeated through the Mag gel column. The particle size distributions of the eluents obtained at various flow rates are presented in Figure 6. At flow rates of 10 and $25 \mathrm{~mL} / \mathrm{h}$, the main particle size in the eluent was $300 \mathrm{~nm}$. After $30 \mathrm{~min}$, silica particles with a wide size distribution were detected in the eluent. This was because during the transport of silica particles through the gaps between the Mag gel particles at low flow rate, the smaller silica particle strongly aggregated and then eluted. At higher flow rate, some of the larger silica particles eluted. Larger silica particles tended to be filtered at the top of the Mag gel layer. However, 
the larger silica particles were more susceptible to the pressure and shear stress from the fluid than the smaller particles, resulting in the elution of some larger particles from the column.

The industrial silica particles remaining in the Mag gel layer after separation were recovered by first collecting the top and bottom halves of the gel layer with a pipette and then by using a magnet to separate the Mag gel from the filtered industrial silica particles. Each suspension was washed with ethanol, as mentioned in Section 2, to shrink the Mag gel particles and remove any adsorbed silica particles on their surface. The obtained solution including separated silica particles was evaluated by DLS, as shown in Figure 7. Silica particles recovered from the top half of the Mag gel layer were large, because this domain contained less deformed Mag gel particles, so it filtered the larger silica particles. However, for the particles obtained from the bottom half of the Mag gel layer, some larger particles were observed because of the enforced transport of smaller silica particles by fluid convection, which induced the aggregation of silica particles. The aggregated silica particles after recovery could possibly be redispersed by solvent addition to weaken the interaction between the silica particles. The above results revealed that the Mag gel layer had the ability to achieve size filtration through deformation in a column, and filtered particles in the Mag gel layer could be recovered by using a magnet to separate the Mag gel and filtered particles.

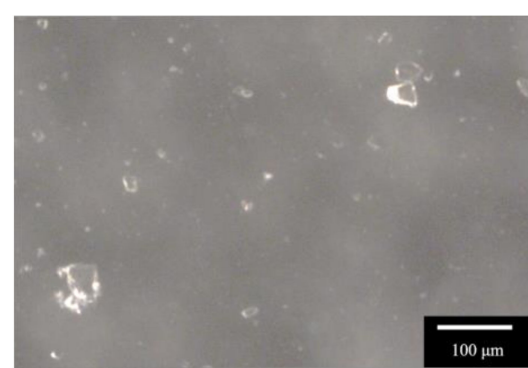

(a)

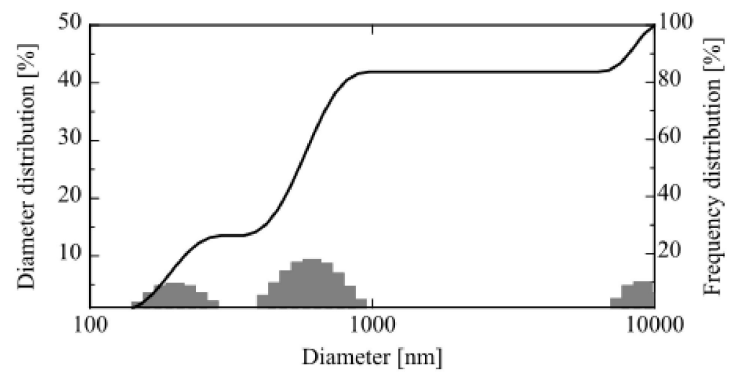

(b)

Figure 5. SEM image (a) and size distribution (b) of the industrial silica particle suspension.

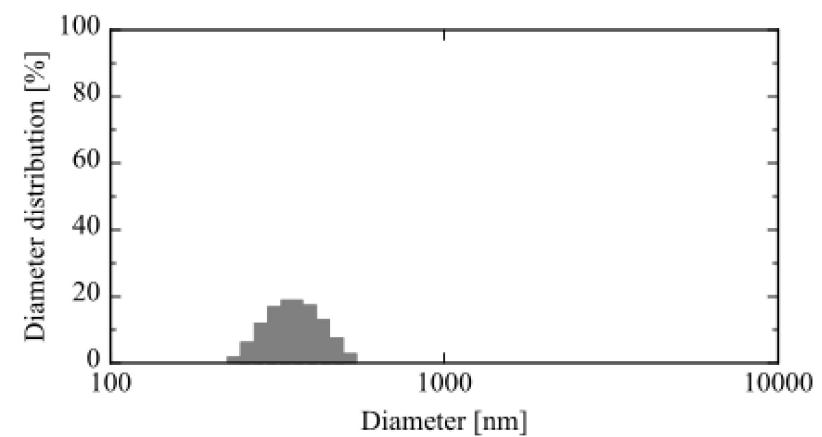

(a)

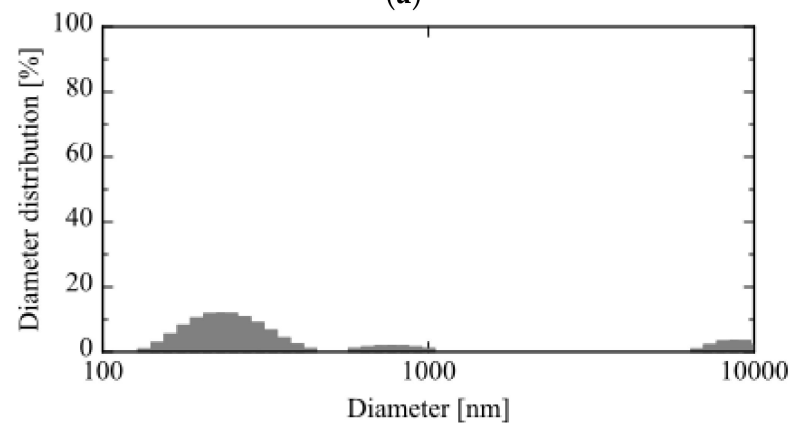

(b)

Figure 6. Cont. 


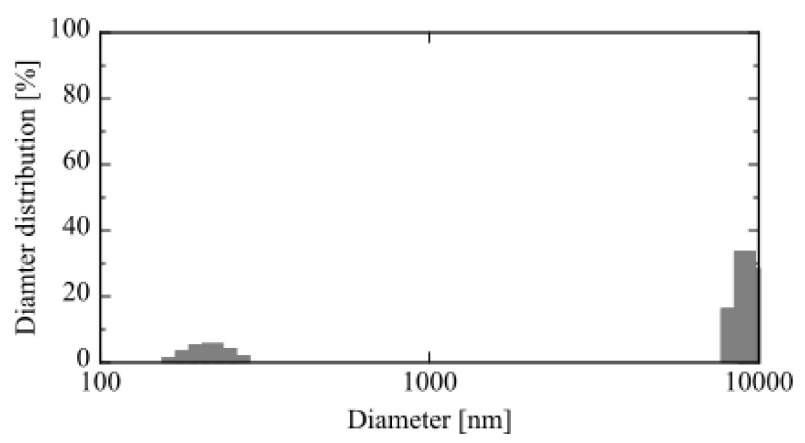

(c)

Figure 6. Size distribution of silica particles eluted from the Mag gel-packed column. (a) Elution time: $15 \mathrm{~min}$, flow rate: $15 \mathrm{~mL} / \mathrm{h}$, (b) elution time: $9 \mathrm{~min}$, flow rate: $25 \mathrm{~mL} / \mathrm{h}$, and (c) elution time: $9 \mathrm{~min}$, flow rate: $50 \mathrm{~mL} / \mathrm{h}$.
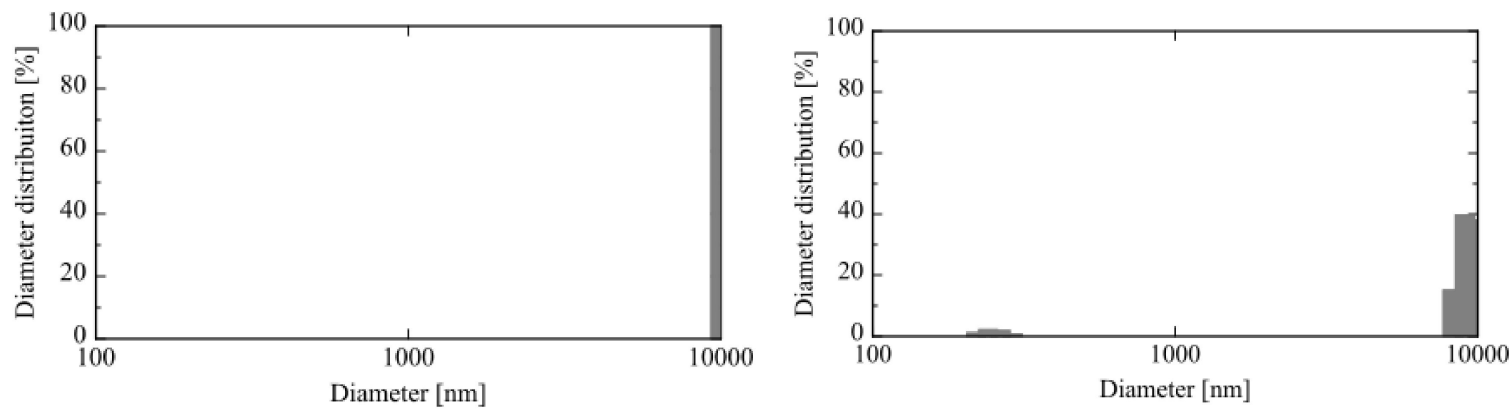

(a) at top domain, flow rate $10 \mathrm{~mL} / \mathrm{h}$

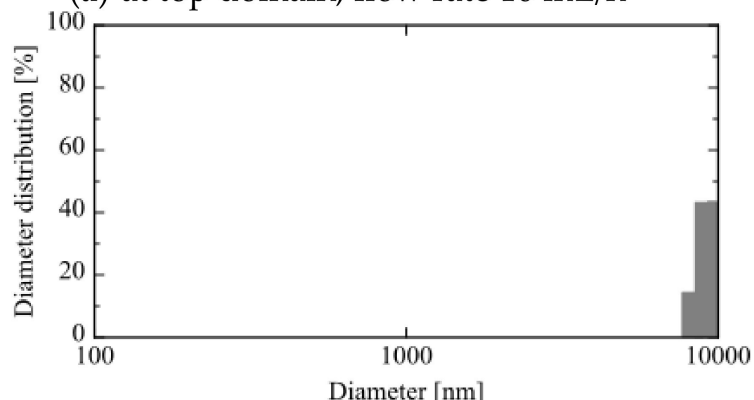

(b) at top domain, flow rate $25 \mathrm{~mL} / \mathrm{h}$

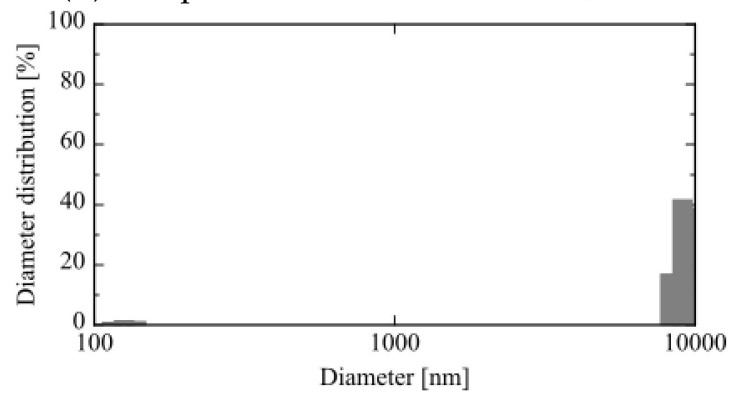

(c) at top domain, flow rate $50 \mathrm{~mL} / \mathrm{h}$

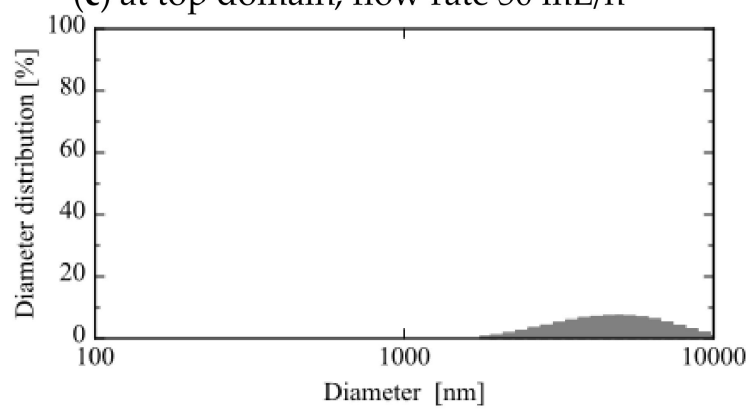

(d) at bottom domain, flow rate $10 \mathrm{~mL} / \mathrm{h}$

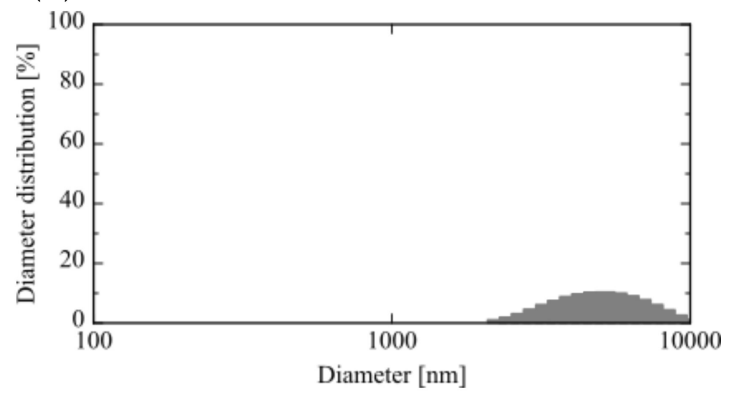

(e) at bottom domain, flow rate $25 \mathrm{~mL} / \mathrm{h}$

(f) at bottom domain, flow rate $50 \mathrm{~mL} / \mathrm{h}$

Figure 7. Size distribution of filtered silica particles obtained from the Mag gel layer, filtered by using different flow rates of water. Silica and Mag gel particles were separated using a magnet. (a-c) Silica particles from the top half of the Mag gel layer and $(\mathbf{d}-\mathbf{f})$ silica particles from the bottom half of the Mag gel layer.

\section{Conclusions}

Magnetite was introduced into spherical gel particles to give a magnetite-containing gel that had the ability to deform under pressure and respond to a magnetic field. Mag gel packed in a column was 
used to separate industrial silica particles with sizes of $300 \mathrm{~nm}, 800 \mathrm{~nm}$, and $10 \mu \mathrm{m}$. A silica particle suspension was injected on the top of the Mag gel layer, and then water was permeated through the layer. The Mag gel layer responded to the pressure and shear stress of the fluid by deforming, causing the gaps between the Mag gel particles to change in the flow direction of the column. The pressure was higher at the bottom of the Mag gel layer than at the top, resulting in smaller gaps between the gel particles at the bottom of the column than at the top. Thus, larger silica particles were captured at the top of the column, and smaller silica particles were filtered to the bottom of the column or eluted. The sizes of the separated silica particles were determined by DLS. The filtered silica particles present in the Mag gel were recovered by the external application of a magnetic field. This separation technique has potential applications for the separation of different silica particles, cells, microorganisms, proteins, and polysaccharides. In engineering, the foundations of gel preparation and column design as well as the operating conditions should be considered in a mathematical manner. The interactions between gel and colloidal particles should also be considered because they affect elution and filtration.

Author Contributions: Conceptualization, M.M. and H.K.; methodology, M.M. and K.Y.; validation, M.M., S.M., K.O., and H.K.; investigation, H.K.; resources, S.M.; data curation, M.M and H.K.; writing-original draft preparation, H.K.; writing-review and editing, H.K.

Funding: This research received no external funding.

Acknowledgments: We thank Natasha Lundin, from Edanz Group (www.edanzediting.com/ac) for editing a draft of this manuscript.

Conflicts of Interest: The authors declare no conflict of interest.

\section{References}

1. Aoi, Y.; Kaneko, Y.; Tsuneda, S. pH-gradient ion-exchange microbial cell chromatography as a simple method for microbial separation. J. Biosci. Bioeng. 2017, 123, 431-436. [CrossRef] [PubMed]

2. Dizge, N.; Soydemir, G.; Karagunduz, A.; Keskinler, B. Influence of type and pore size of membranes on cross flow microfiltration of biological suspension. J. Membr. Sci. 2011, 366, 278-285. [CrossRef]

3. Hwang, K.-J.; Huang, P.-S. Cross-flow microfiltration of dilute macromolecular suspension. Sep. Purif. Technol. 2009, 68, 328-334. [CrossRef]

4. Stegeman, G.; Kraak, J.C.; Poppe, H.; Tijssen, R. Hydrodynamic chromatography of polymers in packed columns. J. Chromatogr. A 1993, 657, 283-303. [CrossRef]

5. Cario, D.D.; Irimia, D.; Tomkins, R.G.; Toner, M. Continuous inertial focusing, ordering, and separation of particles in microchannel. Proc. Natl. Acad. Sci. USA 2007, 27, 18892-18897.

6. Takaoka, Y.; Morisada, S.; Ohto, K.; Kawakita, H. Filtration of colloidal particles using compacted-gel media packed in column. J. Chem. Eng. Jpn. 2017, 50, 815-820. [CrossRef]

7. Takaoka, Y.; Miyoshi, M.; Sakaguchi, K.; Morisada, S.; Ohto, K.; Kawakita, H. Recovery of filtered graphene oxide residue using elastic gel packed in a column by cross flow. Processes 2018, 6, 43. [CrossRef]

8. Ye, E.; Liu, B.; Fan, W.Y. Preparation of graphite-coated iron nanoparticles using pulsed laser decomposition of $\mathrm{Fe}_{3}(\mathrm{CO})_{12}$ and $\mathrm{PPh}_{3}$ in hexane. Chem. Mater. 2007, 19, 3845-3849. [CrossRef]

9. Liu, S.-H.; Gao, H.; Ye, E.; Low, M.; Lim, S.H.; Zhang, S.-Y.; Lieu, X.; Tripathy, S.; Tremelc, W.; Han, M.-Y. Graphitically encapsulated cobalt nanocrystal assemblies. Chem. Commun. 2010, 46, 4749-4751. [CrossRef]

10. Safronov, A.P.; Mikhnevich, E.A.; Lotfollahi, Z.; Blyakhman, F.A.; Sklyar, T.F.; Varga, A.L.; Medvedev, A.I.; Armas, S.F.; Kurlyandskaya, G.V. Polyacrylamide ferrogels with magnetite or strontium hexaferrite: next step in the development of soft biomimetic matter for biosensor applications. Sensors 2018, 18, 257. [CrossRef] [PubMed]

11. Tanaka, H.; Uno, Y.; Morisada, S.; Ohto, K.; Kawakita, H. Filtration and recovery of starch granules using assembled magnetite filter. Chem. Eng. Proc. Proc. Intensif. 2016, 110, 128-133. [CrossRef]

12. Petrova, T.M.; Tzaneva, B.; Fachikov, L.; Hristov, J. Silver recovery from spent photographic solutions by a magnetically assisted particle bed. Chem. Eng. Proc. Proc. Intensif. 2013, 71, 83-96. [CrossRef]

13. Cocker, T.M.; Fee, C.J.; Evans, R.A. Preparation of magnetically susceptible polyacrylamide/magnetite beads for use in magnetically stabilized fluidized bed chromatography. Biotechnol. Bioeng. 1997, 53, 79-87. [CrossRef] 
14. Gerontas, S.; Lan, T.; Micheletti, M.; Tichener-Hooker, N.J. Evaluation of a structural mechanics model to predict the effect of inserts in the bed support of chromatographic columns. Chem. Eng. Sci. 2015, 129, 25-33. [CrossRef]

15. Wu, S.-H.; Mou, C.-Y.; Lin, H.-P. Synthesis of mesoporous silica nanoparticles. Chem. Soc. Rev. 2013, 42, 3862-3875. [CrossRef] [PubMed]

16. Mowla, D.; Karimi, G.; Ostadnezhad, K. Removal of hematite from silica sand ore by reverse flotation technique. Sep. Purif. Technol. 2008, 58, 419-423. [CrossRef] 